\title{
Indy mutations and Drosophila longevity
}

\author{
Blanka Rogina ${ }^{*}$ and Stephen L. Helfand ${ }^{2}$ \\ ${ }^{1}$ Department of Genetics and Developmental Biology, School of Medicine, University of Connecticut Health Center, Farmington, CT, USA \\ ${ }^{2}$ Department of Molecular Biology, Cell Biology and Biochemistry, Division of Biology and Medicine, Brown University, Providence, RI, USA
}

\section{Edited by:}

Elena G. Pasyukova, Institute of Molecular Genetics of Russian Academy of Sciences, Russia

\section{Reviewed by:}

Giovanni Cenci, University of L'Aquila Italy

William Ja, The Scripps Research Institute, USA

\section{${ }^{*}$ Correspondence:}

Blanka Rogina, Department of Genetics and Developmental Biology, School of Medicine, University of

Connecticut Health Center, 263

Farmington Avenue, Farmington, CT 06030-6403, USA.

e-mail:Rogina@neuron.uchc.edu
Decreased expression of the fly and worm Indy genes extends longevity. The fly Indy gene and its mammalian homolog are transporters of Krebs cycle intermediates, with the highest rate of uptake for citrate. Cytosolic citrate has a role in energy regulation by affecting fatty acid synthesis and glycolysis. Fly, worm, and mice Indy gene homologs are predominantly expressed in places important for intermediary metabolism. Consequently, decreased expression of Indy in fly and worm, and the removal of $\mathrm{mlndy}$ in mice exhibit changes associated with calorie restriction, such as decreased levels of lipids, changes in carbohydrate metabolism and increased mitochondrial biogenesis. Here we report that several Indy alleles in a diverse array of genetic backgrounds confer increased longevity.

Keywords: Indy, Drosophila melanogaster, aging and longevity, fruit flies, single gene mutation

\section{INTRODUCTION}

Aging is a complex process that can be modulated by environment and affected by genetic manipulations, such as single gene mutations. Understanding the underlying mechanisms by which single gene mutations extend life span can contribute to our understanding of the process of aging, and allow us to design therapeutic interventions that could postpone age-related decline and extend healthy aging. For example, based on the genetic data shown that down-regulation of the TOR signaling pathway extends longevity of yeast, worms, and fruit flies, experiments were performed that show that rapamycin, a drug that down-regulates the TOR signaling pathway, extends mice and fruit flies longevity (Vellai et al., 2003; Jia et al., 2004; Kapahi et al., 2004; Kaeberlein et al., 2005; Harrison et al., 2009; Bjedov et al., 2010).

Mutations in the Indy (I'm Not Dead Yet) gene extend life span of the fruit fly, Drosophila melanogaster (Rogina et al., 2000; Wang et al., 2009). Similarly, decreased expression of two of the worm Indy homologs extend worm longevity (Fei et al., 2003, 2004). Indy encodes the fly homolog of a mammalian di and tricarboxylate transporter involved in reabsorbing Krebs cycle intermediates, such as citrate, pyruvate, and $\alpha$-ketoglutarate (Knauf et al., 2002, 2006; Pajor, 2006). Functional characterization of the transporter encoded by the Indy structural gene confirmed that it is a transporter of Krebs cycle intermediates (Inoue et al., 2002; Knauf et al., 2002). Studies in frog oocytes and mammalian cells showed that INDY mediates $\mathrm{Na}^{+}, \mathrm{K}^{+}$, and $\mathrm{Cl}^{-}$independent high-affinity flux of dicarboxylates and citrate across the plasma membrane (Inoue et al., 2002; Knauf et al., 2002). Further studies have shown that INDY functions as an anion exchanger of dicarboxylate and tricarboxylate Krebs cycle intermediates (Knauf et al., 2006). Crystal structure of a bacterial INDY homolog from Vibrio cholera (VcINDY) reveals that one citrate and one sodium molecule is bound per protein but the mature transporter is likely found in the form of a dimer (Mancusso et al., 2012).

The fly INDY is most highly expressed in the gut, fat bodies, and oenocytes, all places where intermediary metabolism takes place, suggesting its role in metabolism (Knauf et al., 2002). Similarly, worm homologs (ceNaDC1 and ceNaDC2) are expressed in the intestinal tract (Fei et al., 2003), and the mouse gene mIndy (mINDY; SLC13A5) is predominantly expressed in liver (Birkenfeld et al., 2011). Based on INDY expression and a role in transporting Krebs cycle intermediates it has been hypothesized that decreased INDY activity creates a state similar to calorie restriction (CR). Studies in flies and mice support this hypothesis mainly by showing similarities between the physiology of Indy mutant flies and $m I n d y$ knockout mice on high calorie food and control flies and mice on CR (Wang et al., 2009; Birkenfeld et al., 2011).

It has recently been reported that longevity was not extended in worms with decreased levels of the Indy or in fruit flies with one of the alleles utilized by Rogina et al. (2000) and Toivonen et al. (2007). Toivonen et al. (2007), attributed the life span extension in Indy to the genetic background and bacterial infection (Toivonen et al., 2007). Subsequently, it was confirmed that the original Ind $y^{206}$ mutation extends longevity after backcrossing into the $y w$ background but not after backcrossing into the $w^{1118}$ genetic background (Wang et al., 2009; reviewed in Frankel and Rogina, 2012). Furthermore, it was demonstrated that the results published in Toivonen et al., are most likely due to differences in the caloric content of the food (Toivonen et al., 2007; Wang et al., 2009).

Here we report that the presence of one copy of an Indy ${ }^{206}$ mutant chromosome extends longevity in several genetic backgrounds when compared to genetically matched controls. In order to further address the issues of Wolbachia contamination we treated the previously reported Indy $y^{159}$ allele, and several new 
alleles, with tetracycline and backcrossed all of these Indy alleles into a $y w$ genetic background for 10 generations. We determined survivorships of all Indy alleles on standard laboratory diet and found that several new Indy mutant alleles can also extend the longevity of male and female Drosophila. The data presented here further confirm the role of the Indy gene in Drosophila longevity and show the relationship between life span extension and reduction in Indy mRNA.

\section{RESULTS MUTATION IN Ind $y^{206}$ EXTENDS LIFE SPAN IN DIFFERENT GENETIC BACKGROUNDS}

In order to further examine if genetic background may contribute to the life span extension of heterozygous Indy mutant flies, we determined the survivorship of Indy heterozygous mutant flies in Hyperkinetic ${ }^{1}\left(H k^{1}\right)$ and long- and short-lived selected Luckinbill lines (Figures 1A-E) (Kaplan and Trout, 1969; Luckinbill and Clare, 1985). $H k^{1}$ is a recessive mutation characterized by hyperactivity and shorter life span of Drosophila. Hyperactivity is due to mutation of the beta $\left(H k^{1}\right)$ subunit of the potassium channel, which causes increased neuronal excitability (Trout and Kaplan, 1970). $H k^{1}$ is an X-linked recessive mutation, thus only male flies in those background live shorter (Trout and Kaplan, 1970; Rogina and Helfand, 1995). We used the $H k^{1}$ line since it was isolated by an EMS mutagenesis of Canton-S (CS) stock in 1969 and therefore had many years of divergence from the CS background of the original Indy lines. We determined the survivorship of flies heterozygous for $H k^{1}$ and either Indy ${ }^{206}$ or control-2216. The 2216 and 1085 lines that were derived from the same mutagenesis as Indy ${ }^{206}$, but do not have a P-element insertions in the Indy region were used as control in Rogina et al., 2000. Survivorship analysis revealed that the median life span of male flies with one copy of the Indy mutation in $H k$ background is $52.0 \%$ increased as compared to the control $H k ; 2216$. A similar increase in survivorship of $57.0 \%$ was observed in $H k$; Ind ${ }^{206}$ female flies when compared to the control females, Figures 1A,B; Table 1. (Median life span: $H k ; I n d y^{206}$ males $=38.0$ days, females $=68.0 ; H k ; 2216$ males $=25.0$, females $=43.3$ ).

\section{Indy ${ }^{206}$ MUTANT HETEROZYGOUS FLIES LIVE LONGER IN LUCKINBILL SHORT- AND LONG-LIVED LINES COMPARED TO CONTROL LINES}

Luckinbill short- and long-lived lines were selected based on reproduction of a population of outbreed Drosophila early or late in life (Luckinbill and Clare, 1985). Selective breeding was carried out for 21 or 29 generations and resulted in a large difference in median longevity between short- and long-lived lines. For instance, median life span of males for the short-lived 1S9 line was 33.9 day, while median longevity for the long-lived line $1 L 6=65.3$, 2L9 was 93.0 and $2 L 18$ was 93.0 days. Similar differences in median longevity between short and long-lived line can be seen in females (Median longevity $1 S 9=32.0,1 L 6=61.4$, $2 L 9=80.0$, and $2 L 18=81.0$ days.) We examined if Indy mutant flies can affect longevity of Luckinbill short- and long-lived line differently as compared to controls and further extend the life of long-lived lines beyond that expected from hybrid vigor. Our data show that the Indy ${ }^{206}$ mutation increases longevity of both short- and long-lived lines in all conditions, with one exception, the female 159 ; Ind $y^{206}$ flies have a similar median longevity compared to the controls. While, F1 heterozygous males flies from a cross between the control 1085 and the $1 S 9$ short Luckinbill line show the expected life span extension due to hybrid vigor and have a $77 \%$ increase in median longevity as compared to the homozygous $1 S 9$ line, F1 heterozygous Ind $y^{206}$; $1 S 9$ male flies have much higher increase in median longevity of $98.8 \%$ compared to $1 S 9$ homozygous flies, Figure 1C; Table 1. Ind $y^{206}$ mutation further increased longevity of all long-lived Luckbill lines. F1 heterozygote animals from a cross between the Indy ${ }^{206}$ enhancer-trap line and the laboratory selected long-lived line $1 L 6$ of Luckinbill (Indy ${ }^{206} ; 1 L 6$ ) live $20.7 \%$ longer compared to the homozygous $1 L 6$. In contrast, heterozygous control $1085 ; 1 L 6$, live only $2.5 \%$ longer then homozygous $1 L 6$ flies. 2 L9 homozygous long-lived Luckinbill line live much longer compared to 1L6 Figure 1D. However, Indy mutant heterozygous flies in 2 L9 (Indy ${ }^{206} ; 2 L 9$ ) still live $7.2 \%$ longer compared to the $2 L 9$ homozygous flies Figure 1E. In contrast, F1 heterozygous control males, 1085;2L9 have 4.4\% shorter median life span compared to the homozygous $2 L 9$ flies. Median male life span in days: Indy $y^{206} ; 2 L 9$ males $=99.7,1085 ; 2 L 9=88.9$, Figure 1E; Table 1. Thus, heterozygous Ind $y^{206} ; 2 L 9$ male flies have an increase in life span of $12 \%$ over matched controls (2L9;1085), and $7.2 \%$ over the homozygote Luckinbill long-lived 2L9 line itself (Table 1). Median life span of female 1085;2L9 is decreased by $21.4 \%$ compared to median longevity of homozygous $2 L 9$ female flies, while longevity of Indy ${ }^{206} ; 2 L 9$ females is only $3.2 \%$ shorter compared to homozygous flies. (Median female longevity in days: $1085 ; 2 L 9=62.9$, Ind $y^{206} ; 2 L 9=77.4$, Table 1). Heterozygous Indy ${ }^{206}$ flies in the background of the $2 L 18$ long-lived line do not live significantly longer compared to homozygous $2 L 18$ flies; however, they live significantly longer compared to control 1085; 2 L18 heterozygous male flies, which live 9.5\% shorter compared to $2 L 18$ homozygous male flies. (Median male life span in days: $2 L 18=93.0$, Ind ${ }^{206} ; 2 L 18=94.1,1085 ; 2 L 18=84.2$, Figure 1F; Table 1). Similarly, Indy ${ }^{206} ; 2 L 18$ heterozygous females live longer in $2 L 18$ background compared to the controls.

\section{LIFE SPAN EXTENSIONS IN DIFFERENT Indy ALLELES}

We have previously reported that five independent Indy mutant alleles extend the life span of male and female Drosophila in wild type CS and $y w$ genetic backgrounds (Rogina et al., 2000; Wang et al., 2009). We have now tested an additional six Indy alleles for their effect on fly longevity (Indy ${ }^{E P 3044}$, Indy ${ }^{E P 3366}$, Indy ${ }^{E Y 01442}$, Indy ${ }^{E Y 01458}$, Indy ${ }^{E Y 013297}$, Ind $\left.y^{K G 07717}\right)$. Genomic organization of the Indy locus and position of P-elements insertion in different Indy mutant alleles used in this manuscript is shown in Figure 2A. These six new alleles and three previously tested Indy alleles (Indy $y^{206}$, Ind $y^{302}$, Ind $y^{159}$ ) and $y w$ control flies, were all treated with tetracycline to eliminate any possible bacterial contamination by Wolbachia. Although the absence of Wolbachia contamination after tetracycline treatment was not confirmed by PCR, we have previously confirmed the absence of Wolbachia after identical treatment (Wang et al., 2009). All of the Indy alleles and one of the control stocks 1085, which has the same genetic background as Indy ${ }^{206}$ and Indy ${ }^{302}$, were backcrossed into the $y w$ genetic background for 10 generations. We have determined longevity of all Indy alleles as heterozygotes in 

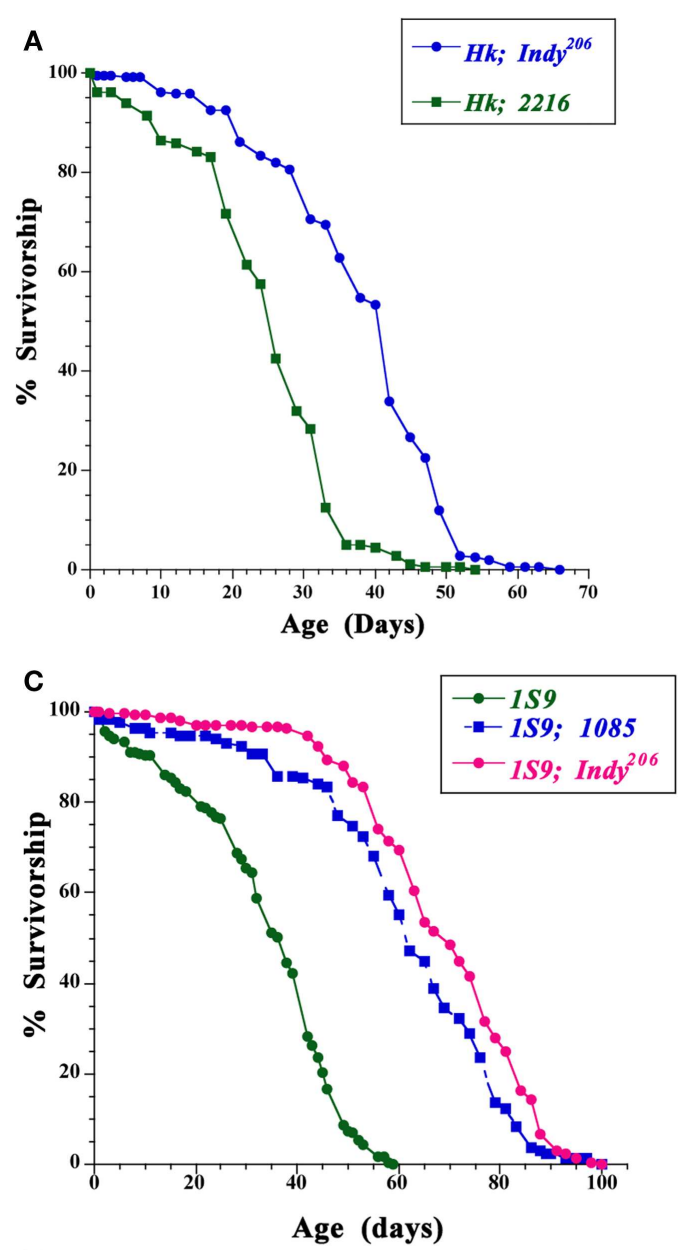

E

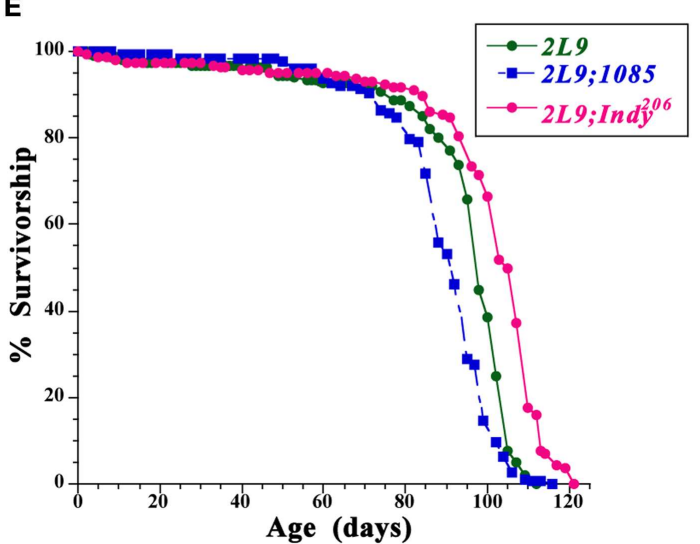

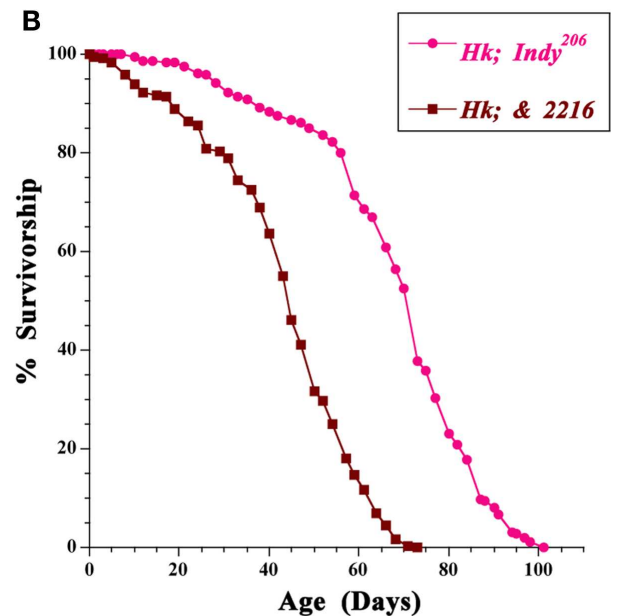

D

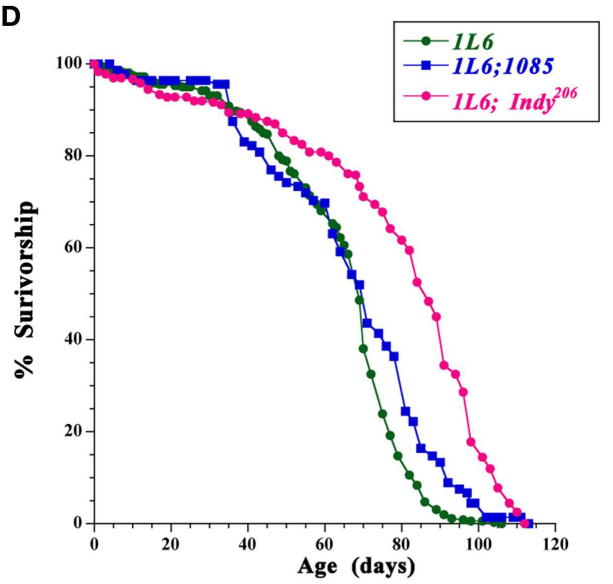

$\mathbf{F}$

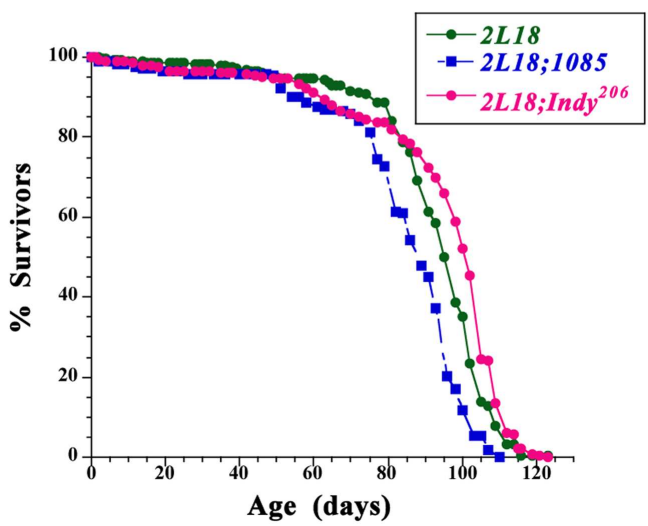

Survivorship for male homozygous flies for the Luckinbill short live line 1S9 (C), the Luckinbill long-lived line 1 L6 (D), 2 L9 (E), or $2 L 18$ (F) and heterozygous for the Indy ${ }^{206}$, or 1085 and the Luckinbill $1 S 9,1 L 6,2 L 9$, or $2 L 18$ lines at $25^{\circ} \mathrm{C}$. Between $135-537$ flies were used for each life span. $y w$ background and calculated median longevity for males and females, Table 2. Representative survivorships of two new Indy alleles are plotted in Figures 2B,C. Heterozygous $y w$;Indy ${ }^{206} /+$, $y w ;$ Ind $y^{302} /+, y w ;$ Ind $y^{159} /+, y w ; I n d y^{E Y 01442} /+y w ; I n d y^{E Y 01458} /+$, and $y w ; I n d y^{E Y 013297} /+$ male and female flies have a significantly longer life compared to control $y w$ flies. Longevity extension in males with one copy of Indy mutant allele varies from 34.4 to $14.0 \%$, and in females Indy mutant extension range from 29.4 to 
Table 1 | Life span of $I n d y^{206}$ heterozygous flies is longer compared to the control flies in different genetic backgrounds.

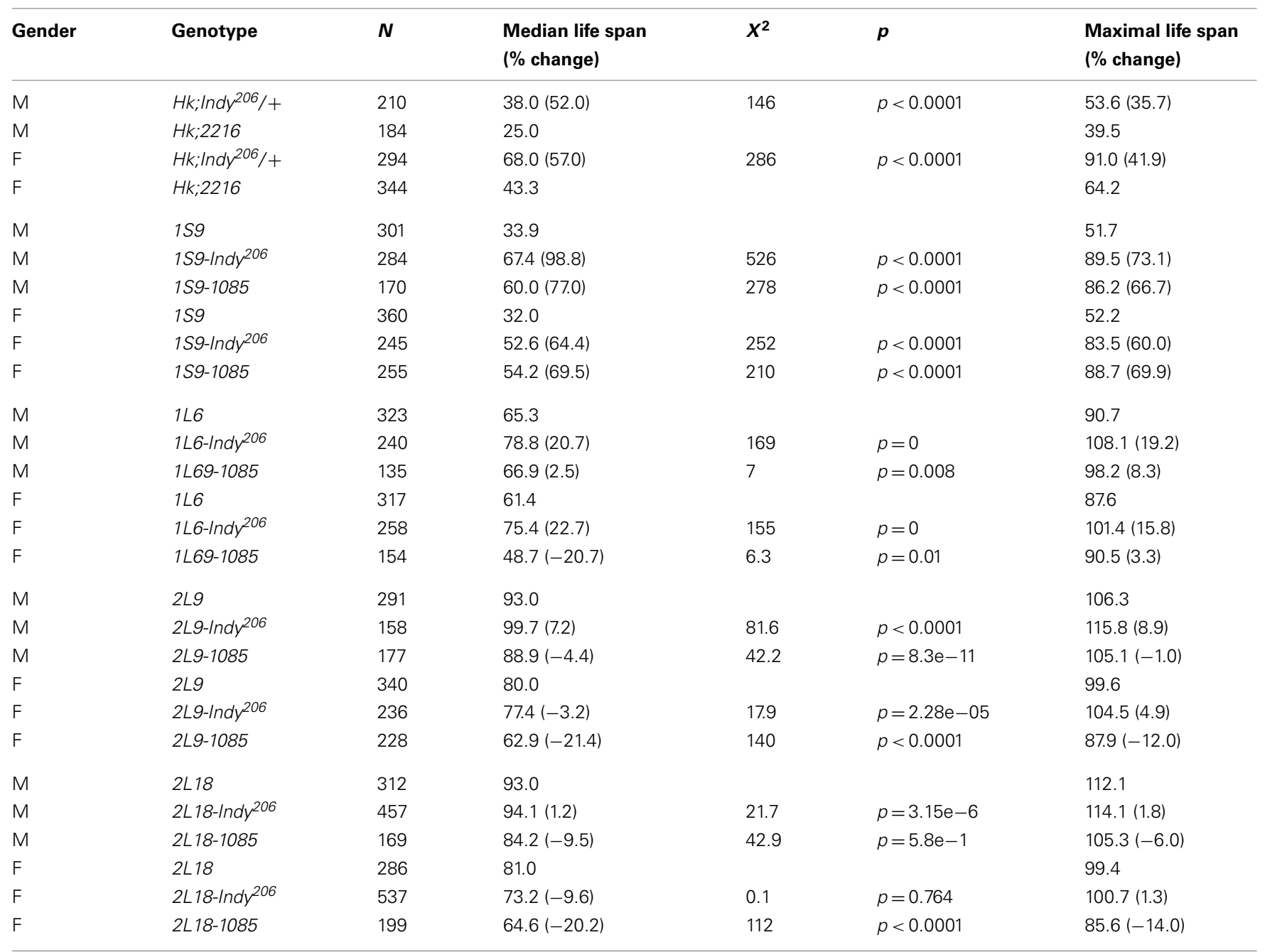

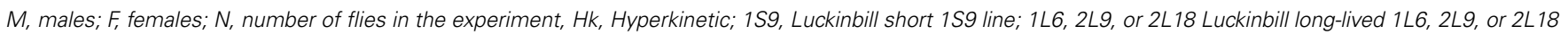
lines.

10.7\%, Table 2. In addition, female, but not male heterozygous $y w$; Ind ${ }^{E P 3044}$ flies live 9.2\% longer compared to the controls. No effect on longevity was observed in male and female heterozygous $y w ;$ Indy $y^{K G 07717} /+, y w ; \operatorname{Ind} y^{3366} /+$, and male heterozygous $y w ;$ Ind $y^{3044} /+$ mutant flies. We determined the levels of Indy mRNA isolated from Head\& Thorax of male heterozygous for two of the new Indy alleles ( $y w$; Ind $y^{E Y 01442} /+, y w$; Ind $\left.y^{E P 3366} /+\right)$, one old $\left(y w ;\right.$ Ind $\left.y^{206} /+\right)$, and their genetic control $(y w)$. The levels of Indy mRNA in heterozygous $y w$; Ind $y^{206} /+$ allele are $51.1 \%$ and in heterozygous $y w$; Ind $y^{E Y 01442} /+$ allele are $60.6 \%$ of the levels of Indy mRNA found in $y w$ flies, Figure 2D. A similar decrease in the levels of Indy mRNA in $y w$; Ind $y^{206} /+$ was previously reported (Wang et al., 2009). We found only a minor, non-significant decrease in the levels of Indy mRNA in heterozygous $y w ; \operatorname{Indy} y^{E P 3366} /+$ mutant flies. Lack of longevity effect in $y w$; Indy ${ }^{E P 3366} /+$ allele is most likely due to only a small effect of the P-element insertion on the Indy mRNA levels in $y w$; Indy ${ }^{E P 3366} /+$ mutant flies. Our data show a strong correlation between the level of Indy mRNA and longevity extension.

\section{DISCUSSION}

We have previously identified and characterized five independent mutations in the Indy gene in Drosophila that cause an increase in average and maximal life span for both male and female fruit flies (Rogina et al., 2000). The original five alleles were derived from three different mutageneses (Boynton and Tully, 1992; Rogina et al., 2000). Life spans of flies carrying one copy of P-element in the Indy gene were compared with their close genetically matched controls, flies from the same mutagenesis without a P-element insertion in the Indy gene. Here we show that Indy ${ }^{206}$ heterozygous mutant flies also live longer when crossed into three different genetic backgrounds, $H k$, short, and long-lived Luckinbill lines as compared to control flies from the same genetic background as Indy also crossed to these three different genetic backgrounds. Luckinbill short and long-lived lines have been generated by selective breeding for early and late female fecundity (Luckinbill and Clare, 1985). Presence of the $y w ;$ Ind $y^{206}$ mutant chromosome significantly extends longevity in the background of the Luckinbill short $1 S 9$ line compared to the control line 1085. Moreover, the 

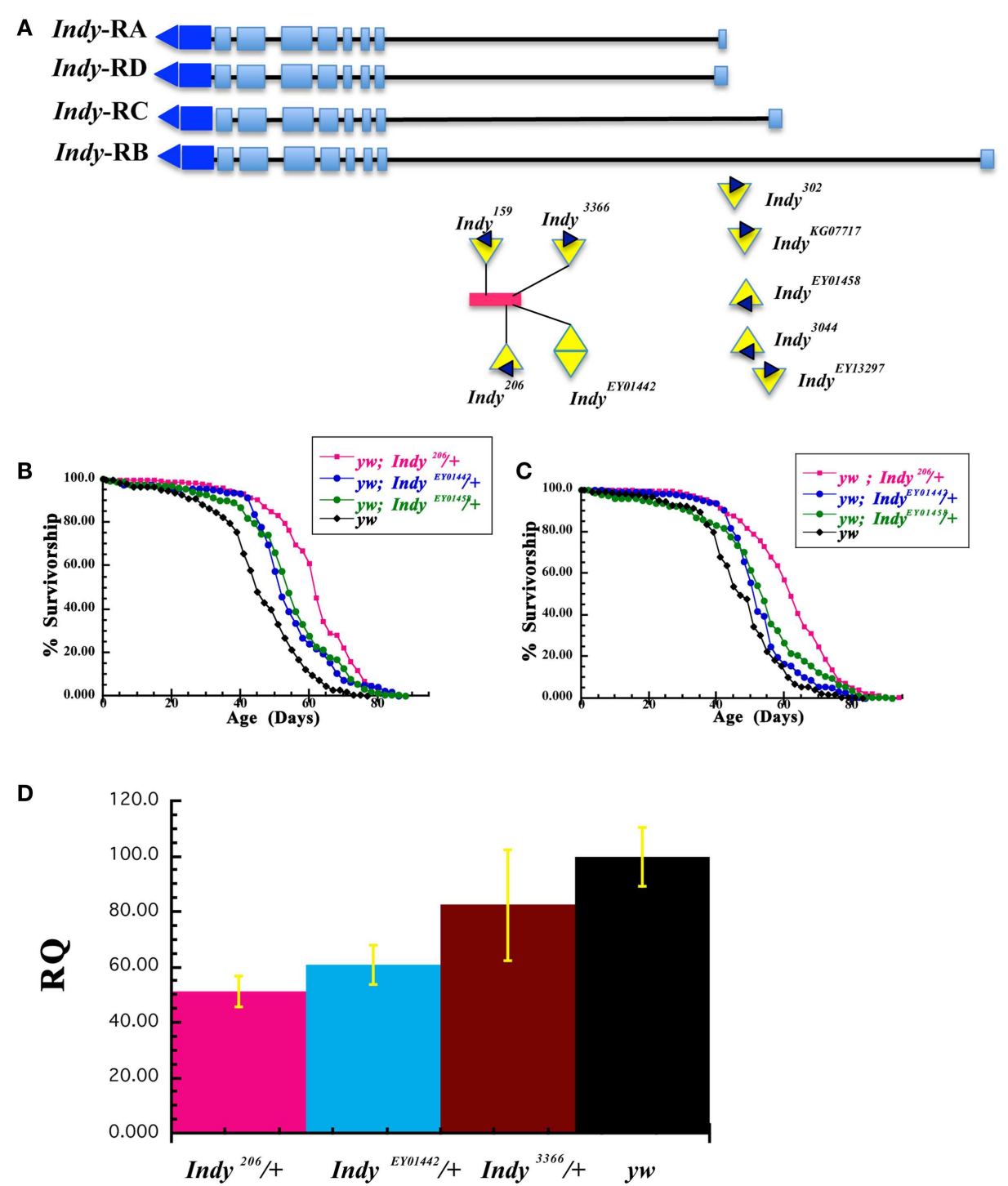

FIGURE 2 | Survivorships of different Indy mutant alleles in $y \boldsymbol{w}$ background. (A) Genomic organization of the Indy locus with insertion sites and orientation of P-element in Ind $y^{206}$, Ind $y^{159}$, Indy $y^{E Y 1442}$, Ind $y^{3366}$, Indy $y^{302}$, Indy $y^{K G 07717}$, Ind $y^{E Y 01458}$, Indy EP3044, and Indy ${ }^{\text {EY013297 }}$ alleles used in

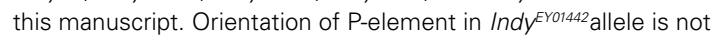
known. The red rectangle represents the conserved Hoppel transposable element. Indy encodes four putative transcripts (RA, RB, $\mathrm{RD}$, and $\mathrm{RC}$ ), which have different $5^{\prime}$ exon. (B,C) Life span of males (B) and females (C) heterozygous for Indy ${ }^{206}$, Indy $y^{\text {EY01442, Indy }}{ }^{\text {EY01458, and } y w}$ on standard laboratory corn diet after $10 \times$ backcrossing into the $y w$. (D) Indy mutants have decreased levels of Indy mRNA. Q-PCR determination of Indy mRNA expression levels in Heads and Thorax of Ind $y^{206} /+$, Indy $y^{\text {EY01442 } /+, ~ I n d ~} y^{33662} /+$, and $y w 20$ days old male flies. Experiments were done in two $\left(/ n d y^{206} /+\right)$ or three $\left(/ n d y^{E Y 01442} /+\right.$, Ind $y^{3366} /+$, and $\left.y w\right)$ replicates with $2 \times 15\left(/ n d y^{206} /+\right), 3 \times 40\left(/ n d y^{3366} /+\right)$, or $3 \times 50\left(/ n d y^{E Y 01442} /+\right.$ and $\left.y w\right)$ flies in each group.
Indy ${ }^{206}$ mutation further extends longevity of two long-lived Luckinbill lines and does not cause shortening of life span of 2 L18 long-lived line. At the same time, median longevity of control lines when crossed to Luckinbill long-lived lines are significantly shorter compared to homozygous Luckinbill lines. These data show that extension of life span by this Indy allele is not limited to the background of the short-lived lines, but further extends lines already selected for long life span.

We also report extension of longevity by additional Indy mutant alleles. All Indy mutant alleles were treated by tetracycline to prevent any effects of Wolbachia and backcrossed to $y w$ background. Wolbachia infection was proposed as a contributing factor to Indy longevity by Toivonen et al. (2007). Indy ${ }^{E Y 01442}$, Ind $y^{E Y 01458}$, Indy ${ }^{E Y 013297}$, Indy ${ }^{K G 07717}$ were generated by the Berkeley Drosophila Genome Project (BDGP) gene disruption project (Bellen et al., 2004). The Indy gene region appears to be a "hot spot" for P-element insertions illustrated by isolation of $5 \mathrm{KG}$, $28 \mathrm{EY}$, and $10 \mathrm{EP}$ element insertions in the Indy region (Bellen et al., 2004). P-element insertion in Ind $y^{206}$, Indy ${ }^{159}$, Indy ${ }^{E Y 01442}$, and Indy ${ }^{E P 3366}$ are within the Hoppel element in the first intron 
Table 2 | Life span of several different Indy mutant alleles as heterozygous is longer compared to the control flies in yw genetic background.

\begin{tabular}{|c|c|c|c|c|c|c|}
\hline Gender & Genotype & $N$ & $\begin{array}{l}\text { Median life span } \\
\text { (\% change to } y w)\end{array}$ & $x^{2}$ & $p$ & $\begin{array}{l}\text { Maximal life span } \\
\text { ( } \% \text { change) }\end{array}$ \\
\hline M & $y w ; \ln d y^{206}$ & 224 & $61.3(34.4)$ & 146 & $p<0.0001$ & 78.9 (19.7) \\
\hline M & $y w ; / n d y^{159}$ & 169 & 54.5 (19.5) & 35.8 & $p=2.16 \mathrm{e}-09$ & $69.2(5.0)$ \\
\hline M & $y w ; I n d y E Y 01442$ & 179 & $53.5(17.3)$ & 30.5 & $p=3.37 \mathrm{e}-08$ & $76.8(16.7)$ \\
\hline M & $y w ; / n d y E Y 01458$ & 151 & $53.8(18.0)$ & 33.5 & $p=6.95 \mathrm{e}-09$ & $75.5(14.6)$ \\
\hline M & $y w ; / n d y K G 07717$ & 178 & $45.9(0.6)$ & 0.9 & $p=0.339$ & $62.5(-5.0)$ \\
\hline M & $y w ; \operatorname{lndy} E P 3044$ & 168 & $48.5(6.3)$ & 1.6 & $p=0.207$ & $75.3(14.3)$ \\
\hline M & $y w ; / n d y=P 3366$ & 181 & $42.8(-6.0)$ & 12.1 & $p=0.000499$ & $57.2(-13.0)$ \\
\hline M & $y w ; 1085$ & 175 & $43.6(-4.0)$ & 5.5 & $p=0.0191$ & $62.3(-5.0)$ \\
\hline M & $y w$ & 169 & 45.6 & & & 65.9 \\
\hline $\mathrm{F}$ & $y w ; I n d y E Y 01442$ & 186 & $52.8(10.7)$ & 11.4 & $p=0.000731$ & $74.1(12.0)$ \\
\hline $\mathrm{F}$ & $y w ; I n d y E Y 01458$ & 206 & $53.0(11.1)$ & 24.5 & $p=8.97 e-07$ & $79.4(20.0)$ \\
\hline $\mathrm{F}$ & $y w ; \operatorname{lndy} E Y 13297$ & 190 & $54.7(14.7)$ & 30.5 & $p=3.36 \mathrm{e}-08$ & $76.1(15.0)$ \\
\hline $\mathrm{F}$ & $y w ; / n d y K G 07717$ & 181 & $48.6(1.9)$ & 0.1 & $p=0.795$ & $70.3(6.3)$ \\
\hline $\mathrm{F}$ & $y w ; \ln d y=P 3044$ & 186 & $52.1(9.2)$ & 7.8 & $p=0.00522$ & $76.9(16.2)$ \\
\hline $\mathrm{F}$ & $y w ; \ln d y=P 3366$ & 189 & $48.9(2.5)$ & 2.4 & $p=0.119$ & $57.3(-13.0)$ \\
\hline $\mathrm{F}$ & $y w ; 1085$ & 187 & $48.4(1.4)$ & 2.2 & $p=0.141$ & $59.2(-11.0)$ \\
\hline $\mathrm{F}$ & $y w$ & 200 & 47.7 & & & 66.2 \\
\hline
\end{tabular}

$M$, males; F, females; $N$, number of flies in the experiment.

of the Indy gene, upstream of the putative translational start site, Figure 2A. The conserved Hoppel element is present in the same position in wild type flies (Rogina et al., 2000). The insertion in Ind $y^{302}, \operatorname{Ind} y^{E Y 013297}$, Ind $y^{E Y 01458}$, Ind $y^{\text {KG07717, }}$, and Indy $y^{E P 3044}$ lines is upstream from putative transcriptional start sites. Indy encodes four putative transcripts, which have different $5^{\prime}$-exons. The positions of P-elements in Indy ${ }^{302}$, Indy ${ }^{E P 3044}$, Indy ${ }^{E Y 01458}$, Ind $y^{E Y 013297}$, and Indy ${ }^{K G 07717}$ are located close to the three putative transcriptional start sites for three putative Indy transcripts (Indy-RA, Indy-RD, and Indy-RC) and about 5,000 bp upstream from the putative transcriptional start site in $I n d y-R B$. Genomic organization of the Indy locus and positions of P-element insertion in different Indy alleles used in this manuscript are shown in Figure 2A. Positions of additional P-elements insertion can be seen in FlyBase: http://flybase.org/reports/FBgn0036816.html. It was previously shown that the presence of the P-element in Ind $y^{206}$ and Indy ${ }^{302}$ mutant alleles decreases the levels of Indy mRNA most likely by affecting transcription (Knauf et al., 2006; Wang et al., 2009). The levels of Indy mRNA are decreased about $95 \%$ in homozygous Ind ${ }^{206}$ and about $40 \%$ in homozygous Indy $y^{302}$ alleles (Wang et al., 2009). The levels of INDY protein are also dramatically decreased in Indy ${ }^{206}$ homozygous mutant flies (Knauf et al., 2002). Similarly, here we show that the levels of Indy mRNA are decreased about 39\% in the heterozygous Indy ${ }^{E Y 01442} /+$ allele and about $49 \%$ in the heterozygous Ind $y^{206} /+$ allele compared to the levels of Indy mRNA found in $y w$ flies. No significant decrease in the levels of Indy mRNA were observed in heterozygous Indy $3366 /+$ flies, which correlates with the absence of longevity extension. It is likely that variation in longevity effects of different Indy alleles correlates to actual Indy mRNA levels and differential effects of P-elements on transcription. We found that male flies heterozygous for six Indy alleles have longevity extension ranging from 14.0 to $34.4 \%$. Females heterozygous for seven Indy alleles show similar result having longevity extension ranging from 9.2 to $29.3 \%$. Our data further confirm our hypothesis that the level of Indy expression is central for longevity extension. When the levels of Indy mRNA are decreased approximately $49 \%$, as in Ind $y^{206} /+$ heterozygous mutant flies, there is dramatic longevity extension of $34 \%$. We have previously reported that when the levels of Indy mRNA are radically reduced, as in Indy ${ }^{206}$ homozygous flies, longevity extension is less than extension of the Ind $y^{206} /+$ heterozygous flies (Wang et al., 2009). A smaller longevity effect of $17 \%$ was observed when Indy mRNA levels are moderately reduced, as in Indy ${ }^{E Y 01442} /+$. Insignificant reduction of Indy mRNA levels, as in Indy ${ }^{E P 3366} /+$ mutant flies, resulted in no longevity effect. Besides Indy ${ }^{E P 3366} /+$, no longevity extension was found in another one of the new alleles, Indy ${ }^{K G 07717}$. In summary, maximal longevity in Indy mutant flies is associated with optimal reduction of Indy mRNA levels. When Indy levels are too low or close to normal, longevity effects are diminished. Although a recent report attributed life span extension in Indy to hybrid vigor, due to life span evaluation in an incorrect genetic background, and bacterial infection, our data presented here corroborate a link 
between the Indy mutations and longevity in flies (Toivonen et al., 2007; Wang et al., 2009). The effect of the Indy mutation on longevity was supported by findings that decreased activity of NaDC2, a C. elegans homolog of the Indy gene, extends the life span of worms (Fei et al., 2003, 2004). Similar effects of increased longevity associated with mutations in the fly and the worm Indy gene suggests a possibility of evolutionary conservation and a universal role of INDY in longevity (Fei et al., 2003, 2004).

Several studies have investigated the molecular mechanisms underlying the effects of the Indy mutation on longevity and health span of worms, flies, and mice (Fei et al., 2003; Marden et al., 2003; Neretti et al., 2009; Wang et al., 2009; Birkenfeld et al., 2011). INDY is a plasma membrane transporter that may mediate the movement of dicarboxylic acids through the epithelium of the gut and into organs important in intermediary metabolism and storage (Knauf et al., 2002, 2006). Location of the INDY transporter in the fat body and oenocytes suggest a role in intermediary metabolism and expression in the gut suggests a role in uptake of nutrients. Reductions in INDY activity may alter uptake, utilization, or storage of important nutrients and affect normal metabolism. It has been hypothesized that reductions in Indy activity seen in Indy mutations might be altering the normal energy supply in flies resulting in life span extension through a mechanism similar to CR. CR has been shown to increase life span and delay the onset of age-related symptoms in a broad range of organisms (McCay et al., 1935; Weindruch and Walford, 1988). Consistent with the hypothesis that Indy is important in metabolism is the finding that Indy mutant worms, flies, and mice have disrupted lipid metabolism (Fei et al., 2003; Wang et al., 2009; Birkenfeld et al., 2011). Similarly to CR animals, Indy mutant flies have increased spontaneous physical activity, decreased starvation resistance, weight, egg production, and insulin signaling. Furthermore, wild type flies on CR have significantly decreased levels of Indy mRNA (Wang et al., 2009). Indy homozygous mutant flies live shorter on low calorie foods compared to controls, which is consistent with our hypothesis that Indy mutant flies are already in a state of reduced nutrition on normal food and when food is further reduced, life span is shortened due to starvation (Wang et al., 2009). In addition, Indy mutant flies have increased mitochondrial biogenesis in heads and thoraces similar to CR animals (Neretti et al., 2009). Similarly, mIndy knockout mice have increased mitochondrial biogenesis in the liver. The mechanism of the effect of a decrease in INDY on metabolism is likely from its physiological function as a citrate transporter. Cytosolic citrate is the main precursor for the synthesis of fatty acid, cholesterol, triacylglycerols, and low-density lipoproteins. In addition, cytosolic citrate inhibits glycolysis and fatty acid $\beta$-oxidation. Therefore, INDY by affecting the levels of cytosolic citrate may alter glucose and lipid metabolism in a manner that favors longevity. Additional support that Indy mutation mimics CR comes from the findings that mIndy knockout mice are protected against adiposity and insulin resistance when kept on high fat diet (Birkenfeld et al., 2011). The data from worm, fly, and mice studies highlight the importance of INDY in health span and longevity. New Indy alleles described here should provide additional tools to further explore the role of INDY in metabolism and its connection to extended longevity and health.

\section{MATERIALS AND METHODS FLY STRAINS}

$1 S 9$ a short-lived and 1L6, 2L9, and 2L18 long-lived lines were a kind gift from James W. Curtsinger and originally described in Luckinbill and Clare (1985). Indy ${ }^{206}$, Indy ${ }^{302}$, 1085, and 2216 were obtained from Tim Tully (Boynton and Tully, 1992). Indy ${ }^{159}$ was kind gift from the Bier lab (Bier

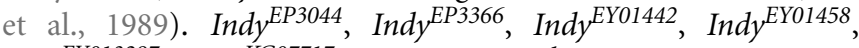
Indy ${ }^{E Y 013297}$, Indy ${ }^{K G 07717}$ alleles, and $H k^{1}$ were obtained from the Bloomington Stock Center or Exelexis. Heterozygous flies used in survivorship analysis are F1 generations from crosses in which virgin females homozygous for $H k^{1}$, short-lived, longlived Luckinbill lines, or $y w$ were mated to males homozygous for different Indy alleles, or the control lines 1085 or 2216.

\section{BACKCROSSING SCHEME}

Indy $y^{206}$, Ind $y^{302}$, Indy ${ }^{159}$, Ind $y^{E P 3044}$, Indy ${ }^{E P 3366}$, Indy $y^{E Y 01442}$, Indy ${ }^{E Y 01458}$, Ind $y^{E Y 013297}$, Indy ${ }^{K G 07717}$, and 1085 were backcrossed into the $y w$ background. Female virgins from $y w$ were mated with males of different Indy alleles or 1085. Heterozygous females were then backcrossed to $y w$ males for 10 generations.

\section{FOOD RECIPE}

We used standard yeast, corn, sucrose food in our experiments: $113 \mathrm{~g}$ Sucrose (MP Biomedicals, Fischer Scientific) and $28 \mathrm{~g}$ Brewers yeast (MP Biomedicals, Fischer Scientific) was mixed with $643 \mathrm{ml}$ water and autoclaved for $20 \mathrm{~min}$. $49 \mathrm{~g}$ corn (MP Biomedicals, Fischer Scientific) and $8.1 \mathrm{~g}$ Agar (SciMart) were mixed in $268 \mathrm{ml}$ water and added to the food mixture and autoclaved for $20 \mathrm{~min}$. The food was cooled down with constant mixing. $2.4 \mathrm{~g}$ tegosept (Fischer Scientific) dissolved in $10.7 \mathrm{ml}$ $100 \% \mathrm{EtOH}$ was added when the food temperature was $65^{\circ} \mathrm{C}$. Approximately $10 \mathrm{ml}$ food was poured to plastic vials using Fly food dispenser (Fischer Scientific), and vials were covered with Kimwipes and cheese cloth. Once the food was cooled down it was stored at $4^{\circ} \mathrm{C}$. Before use the food was warmed up to room temperature.

\section{LIFE SPAN}

Vials were cleared of adult flies in the morning and the collection of newly eclosed flies occurred in the afternoon. Approximately 20 male and 20 female flies were kept together in a plastic vials with approximately $5-10 \mathrm{ml}$ of a standard cornmeal media (Rogina et al., 2000). Flies were housed in humidity-controlled incubators, maintained at $25^{\circ} \mathrm{C}$ on a $12 \mathrm{~h}$ light: dark cycle. Vials of fresh food were supplied three times weekly (Monday, Wednesday, and Friday) and the number of dead flies was recorded during each passage from old to new vials.

\section{mRNA ISOLATION O-PCR ANALYSIS}

The standard Chomczynski protocol and Trizol reagent (Gibco BRL) were used to isolate mRNA (Chomczynski and Sacchi, 1987). Male flies at age 20 were placed on a cold block and Head with Thorax were dissected. Three biological replicates of 50 males were used in each isolations of Ind $y^{E Y 01442} /+$ and $y w$ flies, three 
biological replicates of 40 Indy $y^{3366} /+$ males and two biological replicates of 15 Indy ${ }^{206} /+$ males. Q-PCR was performed with Indy and Ankyrin specific primers obtained from Applied Biosystems according to the manufacturers protocol. Ankyrin was used as an endogenous control. The samples were run on the $\mathrm{AB} 7500$ System.

\section{STATISTICAL ANALYSIS}

Life span data were analyzed by long-rank tests (http://bioinf. wehi.edu.au/software/russell/logrank/). Maximum life span was

\section{REFERENCES}

Bellen, J., Lewis, R. W., Liao, G., He, Y., Carlson, J. W., Tsang, G., et al. (2004). The BDGP gene disruption project: single transposon insertions associated with $40 \%$ of Drosophila genes. Genetics 167, 761-781.

Bier, E., Vaessin, H., Shepherd, S., Lee, K., McCall, K., Barbel, S., et al. (1989). Searching for pattern and mutation in the Drosophila genome with a P-lacZ vector. Genes Dev. 9, 1273-1287.

Birkenfeld, A. L., Lee, H.-Y., GuebreEgziabher, F., Alves, T. C., Jurczak, M. J., Jornayvaz, F. R., et al. (2011). Deletion of the mammalian INDY homolog mimics aspects of dietary restriction and protects against adiposity and insulin resistance in mice. Cell Metab. 14, 184-195.

Bjedov, I., Toivonen, J. M., Kerr, F., Slack, C., Jacobson, J., Foley, A., et al. (2010). Mechanisms of life span extension by rapamycin in fruit fly Drosophila melanogaster. Cell Metab. 11, 35-46.

Boynton, S., and Tully, T. (1992). Latheo, a new gene involved in associative learning and memory in Drosophila melanogaster, identified from P element mutagenesis. Genetics 131, 655-672.

Chomczynski, P., and Sacchi, N. (1987). Single-step method of RNA isolation by acid guanidinium thiocyanate-phenol-chloroform extraction. Anal. Biochem. 162, 156-159.

Fei, Y. J., Inoue, K., and Ganapathy, V. (2003). Structural and functional characteristics of two sodiumcoupled dicarboxylate transporters (ceNaDC1 and ceNaDC2) from Caenorhabditis elegans and their relevance to life span. J. Biol. Chem. 278, 6136-6144.

Fei, Y. J., Liu, J. C., Inoue, K., Zhuang, L., Miyake, K., Miyauchi, S., et al. (2004). Relevance of NAC-2, an $\mathrm{Na}+$-coupled citrate transporter, to life span, body size and fat content in Caenorhabditis elegans. Biochem. J. 379, 191-198.

Frankel, S., and Rogina, B. (2012). Indy mutants: live long and prosper. Front Genet. 3:13. doi:10.3389/fgene.2012.00013

Harrison, D. E., Strong, R., Sharp, Z. D., Nelson, J. F., Astle, C. M., Flurkey, K., et al. (2009). Rapamycin fed late in life extends lifespan in genetically heterogeneous mice. Nature 460, 392-395.

Inoue, K., Fei, Y. J., Huang, W., Zhuang, L., Chen, Z., and Ganapathy, V. (2002). Functional identity of Drosophila melanogaster Indy as a cation-independent, electroneutral transporter for tricarboxylic acidcycle intermediates. Biochem. J. 367, 313-319.

Jia, K., Chen, D., and Riddle, D. L. (2004). The TOR pathway interacts with the insulin signaling pathway to regulate C. elegans larval development, metabolism and life span. Development 131, 3897-3906.

Kaeberlein, M., Powers, R. W. III, Steffen, K. K., Westman, E. A., Hu, D., Dang, N., et al. (2005). Regulation of yeast replicative life span by TOR and $\mathrm{Sch} 9$ in response to nutrients. Science 310, 1193-1196.

Kapahi, P., Zid, B. M., Harper, T., Koslover, D., Sapin, V., and Benzer, S. (2004). Regulation of lifespan in Drosophila by modulation of genes in the TOR signaling pathway. Curr. Biol. 14, 885-890.

Kaplan, W. D., and Trout, W. E. III (1969). The behavior of four neurological mutants of Drosophila. Genetics 61, 399-409.

Knauf, F., Mohebbi, N., Teichert, C., Herold, D., Rogina, B., Helfand, S., et al. (2006). The life-extending gene Indy encodes an exchanger for Krebs-cycle intermediates. Biochem. J. 397, 25-29.

Knauf, F., Rogina, B., Jiang, Z., Aronson, P. S., and Helfand, S. L.

calculated as the median life span of the longest surviving $10 \%$ of the population.

\section{ACKNOWLEDGMENTS}

We thank Suzanne Kowalski and Ryan P. Rogers for their excellent technical help and Dr. Stewart Frankel, Ryan P. Rogers, and Jared Woods for critical reading of the manuscript. This work was supported by a NIA grant AG023088 to Blanka Rogina and by NIA grants AG16667, AG24353, and AG25277 to Stephen L. Helfand.

(2002). Functional characterization and immunolocalization of the transporter encoded by the lifeextending gene Indy. Proc. Natl. Acad. Sci. U.S.A. 99, 14315-14319.

Luckinbill, L. S., and Clare, M. J. (1985). Selection for life span in Drosophila melanogaster. Heredity 55, 9-18.

Mancusso, R., Gregorio, G. G., Liu, Q., and Wang, D. N. (2012). Structure and mechanism of a bacterial sodium-dependent dicarboxylate transporter. Nature 491, 622-626.

Marden, J., Rogina, B., Montooth, K. L., and Helfand, S. L. (2003). Conditional tradeoffs between aging and organismal performance of Indy long-lived mutant flies. Proc. Natl. Acad. Sci. U.S.A. 100, 3369-3373.

McCay, C. M., Crowell, M. F., and Maynard, L. A. (1935). The effect of retarded growth upon the length of life span and upon the ultimate body size. J. Nutr. 10, 63-79.

Neretti, N., Wang, P.-Y., Brodsky, A. S., Nyguyen, H. H., White, K. P., Rogina, B., et al. (2009). Long-lived Indy induces reduced mitochondrial ROS production and oxidative damage. Proc. Natl. Acad. Sci. U.S.A. 106, 2277-2282.

Pajor, A. M. (2006). Molecular properties of the SLC13 family of dicarboxylate and sulfate transporters. Pflugers Arch. 451, 597-605.

Rogina, B., and Helfand, S. L. (1995) Regulation of gene expression is linked to life span in adult Drosophila. Genetics 141, 1043-1048.

Rogina, B., Reenan, R. A., Nielsen, S. P., and Helfand, S. L. (2000). Extended life-span conferred by cotransporter gene mutations in Drosophila. Science 290, 2137-2140.

Toivonen, J. M., Walker, G. A., Martinez-Diaz, P., Bjedov, I., Driege, Y., Jacobs, H. T., et al. (2007). No influence of Indy on lifespan in Drosophila after correction for genetic and cytoplasmic background effects. PLoS Genet. 3:e95. doi:10.1371/journal.pgen.0030095

Trout, W. E., and Kaplan, W. D. (1970). A relation between longevity, metabolic rate, and activity in shaker mutants of Drosophila melanogaster. Exp. Gerontol. 5, 83-92.

Vellai, T., Takacs-Vellai, K., Zhang, Y., Kovacs, A. L., Orosz, L., and Müller, F. (2003). Genetics: influence of TOR kinase on lifespan in C. elegans. Nature 426, 620.

Wang, P. Y., Neretti, N., Whitaker, R., Hosier, S., Chang, C., Lu, D., et al. (2009). Long-lived Indy and calorie restriction interact to extend life span. Proc. Natl. Acad. Sci. U.S.A. 106, 9262-9267.

Weindruch, R., and Walford, R. L. (1988). The Retardation of Aging and Disease by Dietary Restriction. Springfield, IL: C. C. Thomas.

Conflict of Interest Statement: The authors declare that the research was conducted in the absence of any commercial or financial relationships that could be construed as a potential conflict of interest.

Received: 30 November 2012; accepted: 14 March 2013; published online: 08 April 2013.

Citation: Rogina $B$ and Helfand $S L$ (2013) Indy mutations and Drosophila longevity. Front. Genet. 4:47. doi: 10.3389/fgene.2013.00047

This article was submitted to Frontiers in Genetics of Aging, a specialty of Frontiers in Genetics.

Copyright (c) 2013 Rogina and Helfand. This is an open-access article distributed under the terms of the Creative Commons Attribution License, which permits use, distribution and reproduction in other forums, provided the original authors and source are credited and subject to any copyright notices concerning any third-party graphics etc. 